\title{
Is the Use of Frozen Section Indispensable in the Surgical Treatment of Endometrial Hyperplasia?
}

\author{
Ceyhun Numanoglu1 ${ }^{*}$, Dilek Marangoz Chapman², Aysun Fendal Tunca ${ }^{3}$, Aysu Akca3, \\ Agahan Han ${ }^{3}$, Volkan Ulker ${ }^{1}$, Ozgur Akbayir' \\ ${ }^{1}$ Oncology Unit, Department of Obstetrics and Gynecology, Kanuni Sultan Suleyman Training and Research \\ Hospital, Istanbul, Turkey \\ ${ }^{2}$ Ethica Incirli Hospital, Istanbul, Turkey \\ ${ }^{3}$ Department of Obstetrics and Gynecology, Kanuni Sultan Suleyman Training and Research Hospital, Istanbul, \\ Turkey \\ Email: ${ }^{*}$ drceyhun@gmail.com
}

Received 24 March 2015; accepted 10 April 2015; published 14 April 2015

Copyright (C) 2015 by authors and Scientific Research Publishing Inc.

This work is licensed under the Creative Commons Attribution International License (CC BY).

http://creativecommons.org/licenses/by/4.0/

(c) (i) Open Access

\begin{abstract}
Objective: To evaluate the reliability and accuracy of frozen section (FS) compared with final pathology and to determine whether they should be obligatory in every patient with atypical endometrial hyperplasia (AEH). Methods: We retrospectively assessed the medical records of 192 patients whose endometrial biopsy results were endometrial hyperplasia and underwent hysterectomy at our center. Results: Twenty-two (11.4\%) of the 192 patients in our study had concurrent endometrial carcinoma (EC). Of these, 21 (95.5\%) were EC and $1(4.5 \%)$ was non-endometrioid. Of the 140 patients with AEH, 20 (14.3\%) had concurrent EC. Overall, 157 patients had FS. Two (1\%) patients' EC was higher than grade 1B and needed lymphadenectomy. The positive and negative predictive value, specifity and sensitivity of FS were found to be $77.8 \%, 95.7 \%, 97 \%$ and $\mathbf{7 0 \%}$, respectively. Conclusion: Patients who have AEH without high-risk comorbidities do not have to be referred to oncology centers and can undergo hysterectomy in community hospitals without FS.
\end{abstract}

\section{Keywords}

Endometrial Hyperplasia, Coexisting Endometrial Cancer, Atypical Endometrial Hyperplasia, Frozen Section, Hysterectomy

\footnotetext{
${ }^{*}$ Corresponding author.
} 


\section{Introduction}

Endometrial hyperplasia is a well-documented precursor of EC and particularly complex atypical hyperplasia (CAH) where approximately $20 \%$ - 29\% proceed to EC [1]-[4].

Numerous studies have reported that concurrent EC in patients with hyperplasia andatypia ranges from $17 \%$ 62\% [5]-[9]. Chen et al. reported that 54\% of 26 patients with hyperplasia and atypia had cancer at hysterectomy and Hahn et al. found that 10\% of 126 had EC [7] [10]. The difficulties in diagnosing concurrent carcinoma are demonstrated in the lack of interobserver reproducibility in distinguishing between hyperplasia, especially with atypia, and carcinoma [11]-[14]. Preoperative failure to accurately diagnose EC can lead to inadequate staging and potentially suboptimal treatment [15]. If surgery is selected for treatment, the extent of surgery depends on the diagnosis, thus the correleation between pre- and postoperative diagnosis of endometrial pathology comes into prominence [16]. Some authors have suggested the use of perioperative FS because they could play an important role in diagnosis and the decision to extend the surgery.

We retrospectively evaluated the medical records of patients who were preoperatively diagnosed as having $\mathrm{EH}$ and underwent hysterectomy with correlation of FS and final pathology reports. We aimed to determine the requirement for FS and the accuracy of FS compared with final pathology results in patients who underwent surgery for $\mathrm{EH}$, and to evaluate the reliability of the process thereby attempting to determine the most suitable center for treatment, either general gynecology or gyneoncology.

\section{Material and Methods}

The records of 202 patients who underwent hysterectomy for EH at Kanuni Sultan Suleyman Education and Research Hospital, Istanbul, Department of Gynecologic Oncology between January 2002, and December 2013, were evaluated retrospectively. Of the 202 patients, 192 had complete medical and histopathologic records and were included inthe study. Of these 192 patients, 175 were admitted and underwent endometrial biopsy. In the remaining 17 patients, a biopsy was performed because suspicious endometrial thickening was observed during the routine gynecologic examination. Groin pain was recorded as an additional symptom in 12 of the 192 patients (6.25\%). All patients were evaluated with ultrasound scan and none received any type of hormonal treatment. Patient characteristics are shown in Table 1. All patients underwent hysterectomy within 3 months of receiving their histopathologic results.

We evaluated the demographic data, number of pregnancies, presence of menopause, body mass index, findings at admission, histopathology results of preoperative endometrial sampling, FS, and the final histopathology reports. The World Health Organisation (WHO) classification system (1994) for EH was used to classify disease.

Statistical Analyses were performed using SPSS version 20. Continuous data were summarized as mean \pm SD and compared between groups with the use of Mann-Whitney U tests. We chose the Mann-Whitney U test instead of Student's t test becausethe patient number of one of the groups was less than 30. Categorical data was summarized as frequency (\%) and compared between groups using Pearson $\chi^{2}$-tests and Fisher's exact test.

\begin{tabular}{cc} 
Table 1. Patient characteristics. \\
\hline Characteristics \\
\hline Median age & Number \\
Menopause & 51.9 \\
Nulliparity & $63(33 \%)$ \\
Mean Parity & $9(4.7 \%)$ \\
BMI (mean) & 3 \\
DM & 31 \\
Hypertension & $30(15.6 \%)$ \\
Vaginal Bleeding & $50(26 \%)$ \\
\hline
\end{tabular}




\section{Results}

Following are the results of the evaluation of 192 patients.

\subsection{Preoperative Results}

The median age of the patients was 51.9 (37 - 74) years. Nine patients (4.7\%) were nulliparous.In the remaining 183 (95.3\%) patients; the median pregnancy rate was 3 (range, 1 - 12). Sixty-three patients (32\%) were inmenopauseand the remaining 129 (68\%) were in a premenaupausal state. The mean body mass index was 31 ( \pm 5.4 ). Additional comorbidities were diabetes mellitus (DM) and hypertension; 30 patients (15.6\%) had type 2 DM and 50 (26\%) had medically-controlled hypertension.

Preoperative histopathologic results were divided into four categories in accordance with the WHO guidelines; simple hyperplasia without atypia (SNAH), complex hyperplasia without atypia (CNAH), simple endometrial hyperplasia with atypia (SAH), and complex endometrial hyperplasia with atypia (CAH). The distribution was as follows: SNAH 24 patients (12.5\%), CNAH28 (14.6\%), SAH 47 (24.5\%), and CAH 93 (48.4\%) (Table 2).

\subsection{Intra-0perative Results}

Ten (5.2\%) of the 192 patients underwent total abdominal hysterectomy (TAH), 150 (78\%) underwent TAH and bilateral salpingo-oofrectomy (BSO), 16 (8.3\%) underwent total laparoscopic hysterectomy and BSO, 15 (7.8\%) underwent TAH + BSO + lymph node dissection, and 1 (0.5\%) patient underwent vaginal hysterectomy. FS were performed in 157 (81.7\%) patients. Of these 157 patients, 18 (14.5\%) were reported as EC and the remaining 139 (88.5\%) were reported to be benign. The 15 patients who underwent TAH + BSO + lymph node dissection were of the group of 18 patients who were considered to be malignant as a result of the FS. Frozen sections were taken from each of the 140 patients who had simple or CAH, and 17 patients from the CNAH group. Frozen sections were taken from the patients with CNAH due to intra-operative suspicions of malignancy; for example, one patient, aged 51 years, had a suspicious polypoid growth on the fundus, this was diagnosed as malignant both at FS and at final pathology. One cancer from the CNAH group was diagnosed with FS.

\subsection{Postoperative Results}

The final histopathologic examination revealed EC in 14 (77.8\%) ofthe 18 patients who had a malignant FS results. The results of the remaining 4 (22.2\%) patients' final histopathology were benign. The final histopathologic examination resulted with EC in 6 (4.3\%) of the 139 patients who had a benign FS result. From the 35 patients which FS were not performed on, 2 of them diagnosed with CNAH had their final histopathology reported as malignant (Table 3). The positive and negative predictive value, specifity and sensitivity of FSs were found to be $77.8 \%, 95.7 \%, 97 \%$ and 70\%, respectively. The power of FS was calculated at 91.8\% (Table 4).

Table 2. Distribution of patients using the WHO criteria.

\begin{tabular}{ccc}
\hline Preoperative Diagnosis & n & \% \\
\hline Simple endometrial hyperplasia without atypia & 24 & 12.5 \\
Complex endometrial hyperplasia without atypia & 28 & 14.6 \\
Simple endometrial hyperplasia with atypia & 47 & 24.5 \\
Complex endometrial hyperplasia with atypia & 93 & 48.4 \\
Total & $\mathbf{1 9 2}$ & $\mathbf{1 0 0 . 0}$ \\
\hline
\end{tabular}

Table 3. Distribution of patients according to their FS results.

\begin{tabular}{cccc}
\hline Group & Final Histopathology-Malign & Final Histopathology-Benign & Total \\
\hline FS Malign & 14 & 133 & 18 \\
FS Benign & 6 & 33 & 35 \\
No FS & 2 & 170 \\
Total & 22 & 192 \\
\hline
\end{tabular}


The total of 22 patients were diagnosed with endometrium cancer. The final histopathology was adenocarcinoma in 21 patients and adenoacantoma in 1 patient. These patients' disease was classified in accordance with the FIGO staging system; 20 were identified as stage IA disease, one was stage IIA, and one patient was stage IIIC1.

When we evaluated the 93 patients with CAH, 18 (19.4\%) were identified as adenocarcinoma, and of the 140 patients with AEH, 20 (14.3\%) were diagnosed as malignant. 2 patients who were preoperatively diagnosed as having CNAH were confirmed as malignant in the final histopathology. From the CNAH group of 28 patients, 17 had FSandone cancer was diagnosed at FS. This cancer was confirmed in the final pathologic examination.

The age, parity, BMI, comorbid diseases and menopausal state were compared in patients with EC and EH. The menopausal state, hypertension, and meanage were significantly higher in patients with EC (Table 5).

\section{Discussion}

Endometrial hyperplasia is the most common precursor and indicator for the 5th most reported frequent female cancer worldwide in 2012 [17]. Endometrial hyperplasia with atypia is the type most likely to develop into EC, accounting for $>80 \%$ of uterine cancers, despite being the least common type [6].

Rakha et al. found that $38 \%$ of 2550 patients with atypical EH had concurrent carcinoma in their review of 31 studies. There was no difference among the studies where there was a histologic review; however, there was a higher PPV in the studies published after the year 2000 (30\% v 40\%). Similarly, in a review by Trimble et al., the authors reported the frequency of concurrent carcinoma with AEH ranged from $17 \%$ - 52\% in different studies [18].

We performed hysterectomy in 24 patients with simple EH without atypia because they had completed childbearing and had menhoraggia that was resistant to medical therapy. There were nomalignancies reported in their final pathology results. Hysterectomy was also performed for 28 patients with complex EH without atypia. The final histopathologic results of 1 of these patients showed stage IB EC. Due to a very high BMI of 47, one patient's preclinical assessment was suboptimal; however, the patient was observed to have a $3.5 \mathrm{~cm}$-tumor of the cervix that was considered to be an advanced-stage EC. The final histopathologic report showed that it was a stage IIIC EC. Two further patients from the CAH group FSs were diagnosed as being benign and were later confirmed as having cancer.

Our study included 140 patients with atypical EH, of which 20 (14.2\%) had malignant disease. Our results compare to other studies with similarly-sized patient groups, King et al. reported on 119 patients and found 18 cases of concurrent malignancy; Hahn et al. with 126 patients, 13 (10\%) had cancer [7] [19].

Table 4. Frozen section results.

\begin{tabular}{cc}
\hline Frozen section & Results \\
\hline Positive predictive value & $77.8 \%$ \\
Negative predictive value & $95.7 \%$ \\
Specifity & $97 \%$ \\
Sensitivity & $70 \%$ \\
\hline
\end{tabular}

Table 5. Demographic specificities of patients with EH and endometrial carcinoma.

\begin{tabular}{cccc}
\hline & Endometrial Cancer $(\mathrm{n}=22)$ & Endometrial Hyperplasia $(\mathrm{n}=170)$ & P value \\
\hline Age & $55.7 \pm 7.4$ & $51.3 \pm 6.5$ & 0.01 \\
Parity & $3.2 \pm 1.7$ & $3.1 \pm 1.8$ & 0.6 \\
BMI & $32.6 \pm 5.3$ & $30.9 \pm 5.4$ & 0.13 \\
Diabetes n (\%) & $5(22.7)$ & $25(14.7)$ & 0.35 \\
HT n (\%) & $11(50)$ & $42(24.7)$ & 0.006 \\
Menopause n (\%) & $15(68.2)$ & $49(28.8)$ & 0.001 \\
Obesity BMI $\geq 30 \mathrm{n}(\%)$ & $16(72.7)$ & $100(58.8)$ & 0.3 \\
\hline
\end{tabular}


Postmenopausal women with AEH are known to be at the higher risk of EC; women who are premenopausal and have completed childbearing should be treated with total extrafascial hysterectomy [18]. In aggreement with reports that state that older age is associated with EC, we also found that older age increased the risk of cancer; however, the number our patients with cancer was too small to determine a cut-off figure. Suh-Burgmann et al. found a higher frequency in women aged 59 years than in women aged 55 years. In line with the findings of Merissio et al., we found that there was no significant relationship between parity and frequency of cancer [20].

We also found a statistically significant high number of patients with hypertension among those with cancer at 50\%. Eddib et al., in another study found similar results at 64\% although their patient group's mean age was higher at 60.4 years and their BMI was also higher at 34 [21].

There remains some debate regarding the value of intraoperative FS. In the absence of apparent tumor, FSs are typically taken from just two representative samples in which the tumor may not be observed, thus they are only evident at the final histologic examination [20]. Trimble et al. were of the opinion that if hysterectomy is performed for $\mathrm{AEH}$ then intraoperative assessment with FSof the uterine specimen for carcinoma is optional [18]. Conversely, Giuntoli et al. declared that interoperative specimen assessment should be performed; however, the authors conceded that FS often fail to detect EC [22]. Kumar et al. compared intraoperative FS (n = 146) with final pathology in a recent retrospective analysis and found that FS frequently understaged low-risk EC [23]. Shim et al. reported that in 45 cases without apparent myometrial invasion on FS examination, the final histologic analysis revealed the presence of tumor infiltration in $45 \%$ of cases [24].

In contrast with the above findings, 140 patients with AEH and 17 patients with CNAH in our study underwent extrafascial hysterectomy and had FS. Thirty-five patients did not have FS. Of these 35 patients, 2 (5.7\%) were confirmed to have cancer in the final pathology. Among the 157 FS samples, there were 18 (11.5\%) cases reported to be malignant. Of the 18 malignant diagnosis, 14 (77.8\%) were confirmed at final histopathologic examination. The PPV, NPV, specificity, sensitivity and accuracy of FS were 77.8\%, 95.7\%, 97\%, 70\% and 93.6\% respectively. Morotti et al. compared FS and permanent section diagnosis and the concordance between the two techniques was calculated and they showed a substantial agreement. Specificity, sensitivity, NPV, PPV, and accuracy were reported as 93.1\%, 73\%, 73\%, 93.1\%, and 81.8\%, respectively [25]. Even though the NPV and specificity in our study were higher than $90 \%$, our pathologists missed 5 cases of EC, 1 of which was stage IIA. The remaining 4 were low-grade carcinoma that did not require lymphadenectomy or FS.

There is no reliable method to exclude endometrial malignancy intraoperatively and FS have often failed to detect EC [26] [27]. The risk of concurrent high-risk uterine cancer is reported to range from 5\% - 7\% in women whose biopsies are diagnosed as either AEH or endometrial intraepithelial neoplasia [28] [29].

Antonsen et al. advocated that all patients who are diagnosed as having AEH should be treated by specialized gynecological oncologists because $59 \%$ of their 773 patient group had concurrent cancer. While the percentage reported was high, one must also consider their population's median age was 63 years, 80.8\% were postmenopausal, and their median BMI was 27.3 [15]. The median age of our study group was 51.9 years. This wasvery low and was reflected in the percentage of patients with advanced concurrent undiagnosed cancer. Contrary to Antonsen et al. who did not find that BMI was significantly linked to final cancer diagnosis, [15] obesity has been documented as a risk factor for carcinoma. Despite BMI being $31 \mathrm{~kg} / \mathrm{m}^{2}$ in our study, we also found no significant link.

It is not unreasonable to question the validity of FS when one considers the above data. It would be inappropriate to perform lymphadenectomy in all cases as this would result in $90 \%$ - 95\% of patients experiencing unneccesary morbidity and increased risk. While we consider the challenges associated with correct diagnosis and the negative implication of unnecessary procedures, we must find an optimal solution that may be applicable to community centers and specialist unitsalike. We propose that only patients with CAH with one or more risk factors should be considered for FS. Only 1\% of our patients had EC that was more advanced than stage IA. One patient with self-evident stage IIIC cancer had lymphadenectomy; however, a patient with stage IIA EC was misdiagnosed at FS thus failed to have the total required procedure. The vast majority of cases that are indicated for hysterectomy are either benign or at low risk and do not warrant further staging or surgery.

Many patients can undergo surgery at regional centers and without the need for intraoperative FS. Exceptions to this would be patients who have been preoperatively diagnosed as having complex EH, who are at older age, postmenopausal, or with concurrent comorbidities such ashypertension, and patients who cannot be placated and insist on attending an oncology center. This redistribution of patients would significantly reduce the burden on oncology centers. 


\section{Conflict of Interest}

The authors have stated explicitly that there are no conflicts of interest in connection with this article

\section{References}

[1] Kurman, R.J. and Norris, H.J. (1994) Endometrial Hyperplasia and Related Cellular Changes. In: Kurman, R.J., Ed., Blaustein's Pathology of the Female Genital Tract, 4th Edition, Springer Verlag, New York, 411-437. http://dx.doi.org/10.1007/978-1-4757-3889-6 11

[2] Bilgin, T., Ozuysal, S., Ozan, H. and Atakan, T. (2004) Coexisting Endometrial Cancer in Patients with Preoperative Diagnosis of Atypical Endometrial Hyperplasia. Journal of Obstetrics and Gynaecology Research, 30, 205-209. http://dx.doi.org/10.1111/j.1447-0756.2004.00178.x

[3] Salman, M.C., Usubutun, A., Boynukalin, K. and Yuce, K. (2010) Comparison of WHO and Endometrial Intraepithelial Neoplasia Classifications in Predicting the Presence of Coexistent Malignancy in Endometrial Hyperplasia. $J$ ournal of Gynecologic Oncology, 21, 97-101. http://dx.doi.org/10.3802/jgo.2010.21.2.97

[4] Shutter, J. and Wright Jr., T.C. (2005) Prevalence of Underlying Adenocarcinoma in Women with Atypical Endometrial Hyperplasia. International Journal of Gynecological Pathology, 24, 313-318. http://dx.doi.org/10.1097/01.pgp.0000164598.26969.c3

[5] Suh-Burgmann, E., Hung, Y.Y. and Armstrong, M.A. (2012) The Value of Additional Pathology Comments Indicating Suspicion of Adenocarcinoma among Women Diagnosed Preoperatively with Complex Atypical Endometrial Hyperplasia. International Journal of Gynecological Pathology, 31, 222-226. http://dx.doi.org/10.1097/PGP.0b013e31823ef8b0

[6] Rakha, E., Wong, S., Soomro, I., et al. (2012) Clinical Outcome of Atypical Endometrial Hyperplasia Diagnosed on an Endometrial Biopsy: Istitutional Experience and Review of the Literatüre. American Journal of Surgical Pathology, 36, 1683-1690. http://dx.doi.org/10.1097/PAS.0b013e31825dd4ff

[7] Hahn, H.S., Chun, Y.K., Kwon, Y.I., Kim, T.J., Lee, K.H., Shim, J.U., et al. (2010) Concurrent Endometrial Carcinoma Following Hysterectomy for Atypical Endometrial Hyperplasia. European Journal of Obstetrics \& Gynecology and Reproductive Biology, 150, 80-83. http://dx.doi.org/10.1016/j.ejogrb.2010.02.002

[8] Gore, H. and Hertig, A.T. (1966) Carcinoma in Situ of the Endometrium. American Journal of Obstetrics \& Gynecology, 94, 134-155.

[9] Suh-Burgmann, E., Hung, Y.Y. and Armstrong, M.A. (2009) Complex Atypical Endometrial Hyperplasia: The Risk of Unrecognized Adenocarcinoma and Value of Preoperative Dilation and Curettage. Obstetrics \& Gynecology, 114, 523-529. http://dx.doi.org/10.1097/AOG.0b013e3181b190d5

[10] Chen, Y.L., Cheng, W.F., Lin, M.C., Huang, C.Y., Hsieh, C.Y. and Chen, C.A. (2009) Concurrent Endometrial Carcinoma in Patients with a Curettage Diagnosis of Endometrial Hyperplasia. Journal of the Formosan Medical Association, 108, 502-507. http://dx.doi.org/10.1016/S0929-6646(09)60098-X

[11] Giuntoli, R.L. and Zacur, H.A. (2014) Classification and Diagnosis of Endometrial Hyperplasia. Offical Reprint from UpToDate, June 2014.

[12] Giede, K.C., Yen, T.W., Chibbar, R. and Pierson, R.A. (2008) Significance of Concurrent Endometrial Cancer in Women with a Preoperative Diagnosis of Atypical Endometrial Hyperplasia. Journal of Obstetrics and Gynaecology Canada, 30, 896-901.

[13] Kimura, T., Kamiura, S., Komoto, T., Seino, H., Tenma, K., Ohta, Y., et al. (2003) Clinical Over- and UnderEstimation in Patients Who Underwent Hysterectomy for Atypical Endometrial Hyperplasia Diagnosed by Endometrial Biopsy: The Predictive Value of Clinical Parameters and Diagnostic Imaging. European Journal of Obstetrics \& Gynecology and Reproductive Biology, 108, 213-216. http://dx.doi.org/10.1016/S0301-2115(02)00469-4

[14] Mittal, K., Sebenik, M., Irwin, C., Yan, Z., Popiolek, D., Curtin, J., et al. (2009) Presence of Endometrial Adenocarcinoma in Situ in Complex Atypical Endometrial Hyperplasia Is Associated with Increased Incidence of Endometrial Carcinoma in Subsequent Hysterectomy. Modern Pathology, 22, 37-42. http://dx.doi.org/10.1038/modpathol.2008.138

[15] Antonsen, S.L., Ulrich, L. and Hogdall, C. (2012) Patients with Atypical Hyperplasia of the Endometrium Should Be Treated in Oncological Centers. Gynecologic Oncology, 125, 124-128. http://dx.doi.org/10.1016/j.ygyno.2011.12.436

[16] Kleebkaow, P., Maneetab, S., Somboomporn, W., et al. (2008) Preoperative and Postoperative Agreement of Histopathological Findings in Cases of Endometrial Hyperplasia. Asian Pacific Journal of Cancer Prevention, 9, 8991.

[17] World Cancer Research Fund Iternational (2012) http://www.wcrf.org/cancer_statistics/world_cancer_statistics.php

[18] Valenzuela, P., Sanz, J.M. and Keller, J. (2003) Atypical Endometrial Hyperplasia: Grounds for Possible Misdiagnosis 
of Endometrial Adenocarcinoma. Gynecologic and Obstetric Investigation, 56, 163-167. http://dx.doi.org/10.1159/000073950

[19] King, A., Seraj, I.M. and Wagner, R.J. (1984) Stromal İnvasion in Endometrial Adenocarcinoma. American Journal of Obstetrics and Gynecology, 149, 10-14. http://dx.doi.org/10.1016/0002-9378(84)90283-7

[20] Merisio, C., Berretta, R., De, I.A., Pultrone, D.C., Rolla, M., Giordano, G., et al. (2005) Endometrial Cancer in Patients with Preoperative Diagnosis of Atypical Endometrial Hyperplasia. European Journal of Obstetrics \& Gynecology and Reproductive Biology, 122, 107-111. http://dx.doi.org/10.1016/j.ejogrb.2005.01.001

[21] Eddib, A., Allaf, B. and Lee, J. (2012) Risk for Advanced-Stage Endometrial Cancer in Surgical Specimens from Patients with Complex Endometrial Hyperplasia with Atypia. Gynecologic and Obstetric Investigation, 73, 38-42. http://dx.doi.org/10.1159/000329326

[22] Giuntoli, R.L. and Zacur, H.A. (2014) Management of Endometrial Hperplasia. Offical Reprint from UpToDate, June 2014.

[23] Kumar, S., Bandyopadhyay, S., Semaan, A., Shah, J.P., Mahdi, H., Morris, R., et al. (2011)The Role of Frozen Section in Surgical Statingof Low Risk Endometrial Cancer. PLoS ONE, 6, e21912. http://dx.doi.org/10.1371/journal.pone.0021912

[24] Shim, J.U., Rose, P.G., Reale, F.R., et al. (1992) Accuracy of Frozen Section Diagnosis at Surgery in Clinical Stage I and II Endometrial Carcinoma. American Journal of Obstetrics and Gynecology, 166, 1335-1338. http://dx.doi.org/10.1016/0002-9378(92)91600-F

[25] Morotti, M., Menada, M.V., Moioli, M., Sala, P., Maffeo, I., Abete, L., Fulcheri, E., Menoni, S., Venturini, P. and Papadia, A. (2012) Frozen Section Pathology at Time of Hysterectomy Accurately Predicts Endometrial Cancer in Patients with Preoperative Diagnosis of Atypical Endometrial Hyperplasia. Gynecologic Oncology, 125, 536-540. http://dx.doi.org/10.1016/j.ygyno.2012.02.011

[26] Indermaur, M.D., Shoup, B., Tebes, S. and Lancaster, J.M. (2007) The Accuracy of Frozen Pathology at Time of Hysterectomyin Patients with Complex Atypical Hyperplasia on Preoperative Biopsy. American Journal of Obstetrics and Gynecology, 196, e40-e42. http://dx.doi.org/10.1016/j.ajog.2006.10.886

[27] Attard Montalto, S., Coutts, M., Devaja, O., Summers, J., Jyothirmayi, R. and Papadopoulos, A. (2008) Accuracy of Frozen Section Diagnosis at Surgery in Pre-Malignant and Malignant Lesions of the Endometrium. European Journal of Gynaecological Oncology, 29, 435-440.

[28] Valenzuela, P., Sanz, J.M. and Keller, J. (2003) Atypical Endometrial Hyperplasia: Grounds for Possible Misdiagnosis of Endometrial Adenocarcinoma. Gynecologic and Obstetric Investigation, 56, 163-167. http://dx.doi.org/10.1159/000073950

[29] Beutler, H.K., Dockerty, M.B. and Randall, L.M. (1963) Precancerous Lesions of the Endometrium. Obstetrical \& Gynecological Survey, 18, 987-991. http://dx.doi.org/10.1097/00006254-196312000-00048 